\title{
An LMI Approach to IMC-Based Robust Tunable Control
}

\author{
Benoit Boulet, Yingxuan Duan and Hannah Michalska \\ Centre for Intelligent Machines, McGill University \\ 3480 University Street, Montréal, Québec, Canada H3A 2A7
}

\begin{abstract}
This paper presents a linear matrix inequalities (LMI) approach to robust tunable control. This controller design technique provides a new on-line tuning strategy for industrial process control systems. The tuning strategy is based on the performance robustness bounds of the system and knowledge of the plant uncertainty's weighting function, which may change with time. The internal model control structure of the controller is adopted together with additive plant uncertainty. The design and tuning of the robust tunable controller for an SISO system relies on an approximate solution to the two-disc optimization problem, that is solved over the class of discrete-time finite impulse response filters via the solution of an LMI problem. A numerical example is given to illustrate the technique.
\end{abstract}

\section{Introduction}

For linear time-invariant plants, model uncertainty can often be characterized as unknown stable perturbations, bounded by the magnitudes of given weighting functions in the frequency domain. The following controller design question arises from robustness considerations: Given a model with uncertainties, what is the best possible controller design that will optimize some performance criterion for the worst-case model in the set? Typically, a robust $\mathcal{H}_{\infty}$ or $\mu$ controller design is based on a set of weighting functions representing performance specifications and uncertainty sets with the goal of achieving the best robustness/performance tradeoff. Once the robust controller is implemented, its parameters are fixed and no tuning is possible. This may result in performance degradation or even instability as the plant dynamics change over time, e.g., from system component wear, or from changes in the raw material properties of industrial processes. Thus, the capability of tuning the controller is often required in order to trade-off performance and robustness on-line since an initial $\mathcal{H}_{\infty}$ or $\mu$ controller design rarely has the best possible weighting functions for the plant [3].

Thus, as mentioned in [10], an important new challenge for control research is to provide industry with a set of design techniques and implementation tools for robust, on-line tunable controllers. It can be argued that one of the reasons why decentralized single-input single-output PID control is still widely used in the process industry (despite potentially severe performance limitations), is that PID controllers can be tuned on-line by operators or plant engineers to maintain process performance after a change in dynamics.
Internal model control (IMC) [2] is an attractive approach in that it allows some tuning of the IMC filter $Q$ while keeping the nominal closed loop stable. IMC is based on the parameterization of all stabilizing controllers for stable plants: $K=(I-Q P)^{-1} Q$, where $Q \in \mathcal{R} \mathcal{H}_{\infty}$, and where $P$ is the nominal plant model.

Following our preliminary results on robust tunable control for SISO systems in [10], this paper presents a linear matrix inequality (LMI) strategy to design and tune a robust tunable controller. As shown in [10], IMC-based robust tunable control hinges on the 'two-disc' optimization problem. For stable SISO systems, the solution to the twodisc problem was given in terms of the optimal frequency response of the IMC filter. When the size of the uncertainty changes, the IMC filter should be retuned according to the new weighting function to achieve the best robust performance tradeoff. An approach to retuning an FIR IMC filter on-line via an LMI optimization is given.

One of the main goals of feedback control is to maintain system performance despite the presence of possibly timevarying plant uncertainty. Using mu-synthesis via a $\mathrm{D}-\mathrm{K}$ iteration is a systematic and often successful approach for achieving this objective. But there are known problems with the D-K iteration procedure. The most pronounced problem is that it does not always converge to a global minimum. Even if both the K-step and D-step are convex they are not jointly convex. In principle, the scaled $\mathcal{H}_{\infty}$ norm should decrease at each step with reasonable computational complexity, but in practice the effectiveness of D-K iteration depends on the quality of fit of the Dscales. There is a tradeoff between fitting quality and order of the D-scales. With a poor fit, the scaled $\mathcal{H}_{\infty}$ norm can even increase in subsequent iterations. So, for an on-line controller redesign scheme, the computational cost of D-K iteration may be too high. Furthermore, the execution of the algorithm often needs to be overseen by an engineer to steer it out of numerical pitfalls.

On the other hand, the strategy that we introduce here gives the designer another way to approach the on-line tuning of the controller. It uses an LMI approach to get an FIR IMC filter which approximately matches the optimal frequency response. As the numerical example shows, the results have a reasonable computational cost and good convergence. This technique could support automatic or operator-initiated on-line controller tuning according to uncertainty changes or new performance requirements for the plant. 


\section{Theory of IMC Based Robust Tunable Control}

\subsection{Background}

The concept of Robust Tunable Control was introduced in [10] through the construction of an equivalent IMC block diagram to the general unity-feedback control system [2]. Consider the IMC structure in Figure 1. It is well known that for a stable plant, nominal closed-loop stability is guaranteed iff the IMC filter $Q$ is stable. Here, we consider a nominal plant $P$ with uncertainty $\Delta_{a}$ whose size is specified by the scalar weighting function $W_{a}$. For robust performance, there is a performance weight $W_{p}$ on the error signal (for sensitivity minimization) and a weight $W_{u}$ is placed on the control signal to satisfy actuator constraints (see Figure 1.) We pull out the normalized additive perturbations and rearrange the system into a $G-\Delta_{a}$ linear fractional transformation (LFT) form, as shown in Figure 2(a).

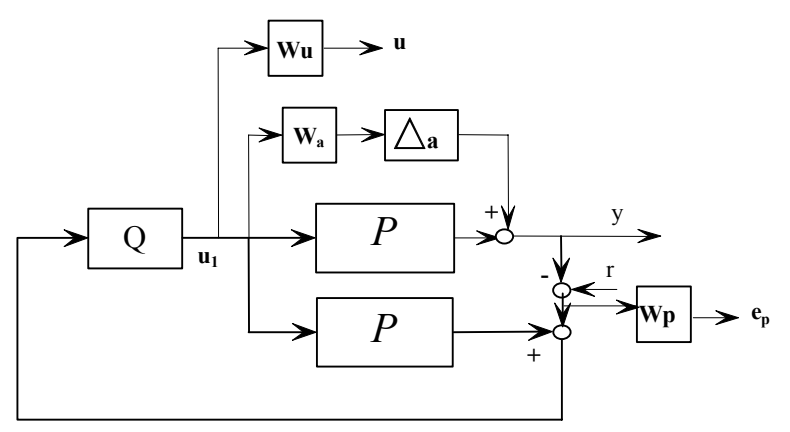

Figure 1: IMC block diagram with weighting functions

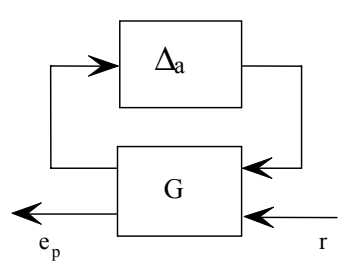

Figure 2(a)

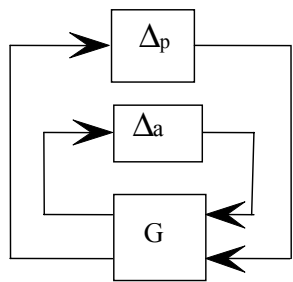

Figure 2(b)
Figure 2: (a) LFT form of system; (b) Setup for $\mu$-analysis

Robust performance of the system requires that the upper linear fractional transformation $\mathcal{F}_{U}\left\{G, \Delta_{a}\right\}$ satisfy $\left\|\mathcal{F}_{U}\left\{G, \Delta_{a}\right\}\right\|_{\infty} \leq 1, \forall\left\|\Delta_{a}\right\|_{\infty}<1$. This can be tested by computing the structured singular value $\mu_{\Gamma}[G(j \omega)]$ at all frequencies, where $\Gamma:=\left\{\operatorname{diag}\left\{\Delta_{a}, \Delta_{p}\right\}: \Delta_{a} \in \mathbb{C}, \Delta_{p} \in \mathbb{C}\right\}$ is the uncertainty structure. The transfer matrix $G$ is given by:

$$
G=\left[\begin{array}{cc}
-W_{a} Q & W_{a} Q \\
-W_{p}(I-Q P) & W_{p}(I-Q P)
\end{array}\right]=:\left[\begin{array}{ll}
G_{11} & G_{12} \\
G_{21} & G_{22}
\end{array}\right]
$$

The robust performance condition is satisfied iff $\sup \mu_{\Gamma}[G(j \omega)] \leq 1$. This is satisfied iff, for each frequency [1],

$$
\mu_{\Gamma}[G]=\inf _{d_{\omega} \in \mathbb{R}_{+}} \bar{\sigma}\left(\left[\begin{array}{cc}
\left\|G_{11}\right\| & d_{\omega}\left\|G_{12}\right\| \\
\frac{1}{d_{\omega}}\left\|G_{21}\right\| & \left\|G_{22}\right\|
\end{array}\right]\right) \leq 1 .
$$

The "two-block $\mu$ " has the following upper bound:

$$
\begin{aligned}
\mu_{\Gamma}[G] & \leq \sqrt{\left\|G_{11}\right\|^{2}+\left\|G_{22}\right\|^{2}+2\left\|G_{12}\right\|\left\|G_{21}\right\|^{2}} \\
& =\left\|W_{a} Q\right\|+\left\|W_{p}(I-Q P)\right\|
\end{aligned}
$$

Thus, a sufficient condition for robust performance is provided by the following upper bound:

$\mu_{\Gamma}[G] \leq\left\|W_{a} Q\right\|+\left\|W_{p}(I-Q P)\right\| \leq 1, \quad \forall \omega$.

This sufficient condition is actually also necessary for an SISO plant, i.e., the upper bound on $\mu_{\Gamma}[G(j \omega)]$ is tight [1].

Since we want to keep the stable IMC filter $Q$ tunable, there are two questions that arise: Given the weighting functions, what is the optimal $Q$ that would minimize the upper bound on $\mu_{\Gamma}[G]$ ? And, what is the range and best "direction" of tuning for $Q$ so that the upper bound remains less than one? Suppose the plant is square, $n \times n$. To answer the first question, a minimization problem is set up frequency by frequency:

$$
\min _{\|Q\|\left\langle\left|W_{a}\right|^{-1}, Q \in \mathbb{C}^{n \times n}\right.}\left\|W_{a} Q\right\|+\left\|W_{p}(I-Q P)\right\| .
$$

where all transfer functions are evaluated at $s=j \omega$ and the constraint $\|Q\|<\left|W_{a}\right|^{-1}$ enforces robust stability. This is the so-called two-disc optimization problem in $\mathcal{H}_{\infty}$ control theory.

\subsection{Two-disc Problem}

A theory for the two-disc problem has been developed in [5],[6] by expressing the problem as a distance minimization in a certain Banach space, and then applying Banach space duality methods to characterize the solution. The theory in [5],[6] leads to a precise characterization of the solutions as well as a numerical approximation. It is proved that:

1- An optimal $Q$ which minimizes (7) does exist under the following assumptions : a) Plant $P$ is strictly proper; b) $W_{a}, W_{p}$ are continuous. The result of the minimization is a 
function of the weighting matrices and is restricted to a certain range.

2- The optimal $Q$ can be approximated by a finite convex optimization in the following manner: Restrict $Q$ to lie in the space $\mathcal{P}_{m}$ consisting of degree $m$ analytic polynomials of the form $a_{0}+a_{1} z+\ldots+a_{m} z^{m}$ with real coefficients, and then discretize the unit circle sufficiently finely with respect to $m$. This yields a convex problem in the variables $a_{0}, a_{1}, \ldots, a_{m}$. For any fixed $m$, these convex problems generate upper bounds for $\mu$ and suboptimal control laws, since $Q$ is restricted to a proper subspace of $\mathcal{H}_{\infty}$. Such problems are then amenable to standard applications of convex programming techniques. Such a technique is the ellipsoid algorithm of Shor, Yudin and Nemirovski, which can solve the problem approximately in the framework of a Linear Matrix Inequality Problem [9]. We use a similar approach of an LMI formulation of the robust performance problem in the numerical example.

\subsection{Tuning for SISO plants}

For SISO plants, the sufficient condition for robust performance in (4) is also necessary as the bound is tight:

$\mu_{\Gamma}[g]=\left|w_{a} q\right|+\left|w_{p}(1-q p)\right| \leq 1, \quad \forall \omega$

In this section, we provide answers to the two questions posed above for minimum-phase SISO plants. The above minimization problem can be reformulated as follows:

$F=\gamma \min _{q_{1} \in \mathbb{C},\left|q_{1}\right| \leq \gamma^{-1}}\left\{\left|q_{1}\right|+\beta \gamma^{-1}\left|1-q_{1}\right|\right\}$

where $q_{1}:=q p \in \mathbb{C}, \quad \gamma:=\left|w_{a}\right||p|^{-1}$ is the relative size of the uncertainty, and $\beta=\left|w_{p}\right|$. The quantity $\left|q_{1}\right|+\beta \gamma^{-1}\left|1-q_{1}\right|$ is best viewed as the sum of lengths of vectors $q_{1}$ and $\beta \gamma^{-1}\left(1-q_{1}\right)$ in the complex plane. The vector $\beta \gamma^{-1}\left(1-q_{1}\right)$ starts from the tip of vector $q_{1}$ and passes through the point 1 , as shown in Figure 3. It is clear that the minimum sum of vector lengths is obtained when the two vectors are aligned on the positive real axis. This means that the best direction for tuning the IMC filter $q$ is along the inverse of the nominal plant.

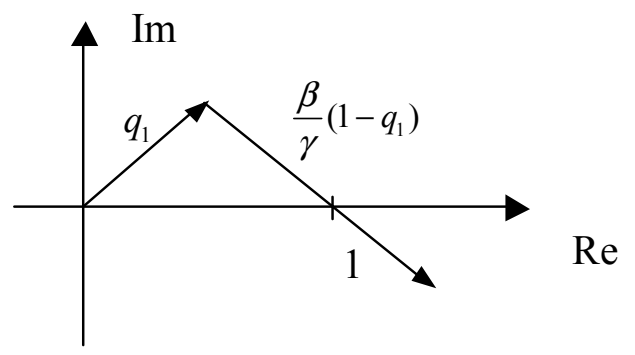

Figure 3: Minimization of the sum of two vector lengths
From Figure 3, we have the following cases leading to different solutions to (7), all based on the required alignment property of vectors $q_{1}$ and $\beta \gamma^{-1}\left(1-q_{1}\right)$ on the real line to minimize their sum of lengths:

Case $\gamma=\beta$ : the relative uncertainty is equal to the performance spec. Then, optimal $q_{\text {lopt }} \in\left[0, \min \left\{1, \gamma^{-1}\right\}\right] \subset \mathbb{R}$, and $F=\gamma$.

Case $\beta>\gamma$ : the performance spec is greater than the relative uncertainty. Then, $q_{1 \text { opt }}=\min \left\{1, \gamma^{-1}\right\}$,

$$
F=\left\{\begin{array}{cc}
\gamma, & q_{1 o p t}=1 \\
1+\beta\left(1-\gamma^{-1}\right), & q_{1 o p t}=\gamma^{-1}
\end{array} .\right.
$$

Case $\beta<\gamma$ : the performance spec is less than the relative uncertainty. Then, $q_{1 \text { opt }}=0$, for which $F=\beta$.

Note that the optimal $q_{\text {lopt }}$ is real in each case. These results stated in terms of the nominal plant model and the weighting functions are summarized in Theorem 1 below.

Theorem 1:

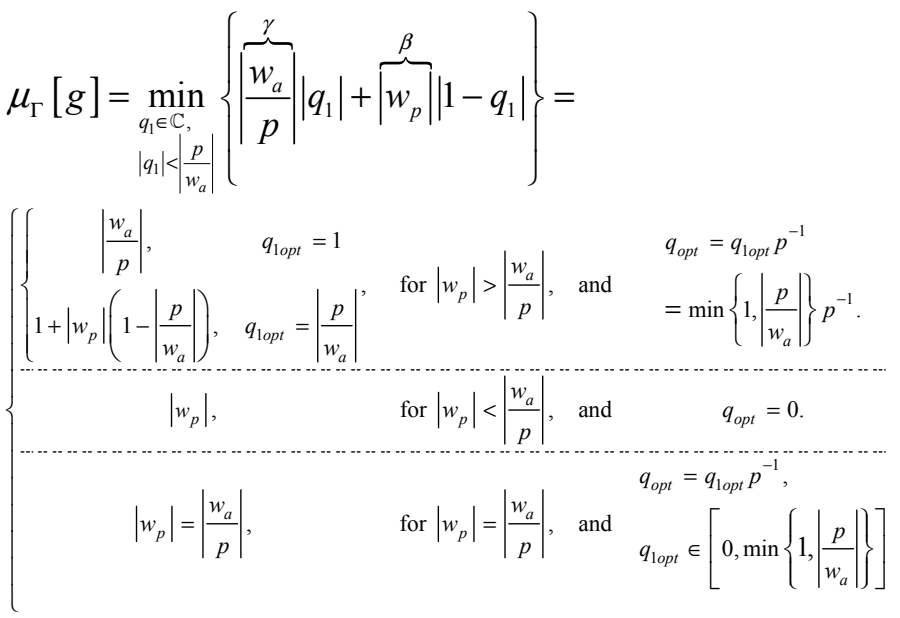

Where $q_{o p t}$ is the optimal frequency response of the IMC filter that minimizes $\mu_{\Gamma}[g]$.

\section{Remarks:}

1- The real number $q_{1 \text { opt }}$ can be seen as a gain on $q_{\text {opt }}$. The most favorable direction of the IMC filter $q$ for improving robust performance is along the inverse of the plant. That is, the phase of $q(j \omega)$ should be set equal to the phase of $p^{-1}(j \omega)$.

2- When the performance weight exceeds the relative uncertainty, the robust performance index $\mu_{\Gamma}[g]$ cannot go below the latter. This is a typical situation in the passband of the control system. In this case, the optimal 
IMC filter is the plant inverse, which is of course impractical as it leads to a controller with infinite gain at frequency $\omega$.

3- When the performance weight is smaller than the relative uncertainty, the robust performance index $\mu_{\Gamma}[g]$ is limited by the former. This is a situation where the uncertainty dominates the tradeoff, e.g., at high frequencies. It turns out that the optimal robust performance level $\mu_{\Gamma}[g]=\left|w_{p}\right|$ is obtained by "turning off" the controller at frequency $\omega$, which of course leads to a direct open-loop path from the reference through the weighting function $w_{p}$.

4- Finally, when the relative uncertainty is equal to the performance weight in magnitude, all IMC filters on a straight line between 0 and the plant inverse are optimal.

5- A necessary condition for robust performance is: $\min \left\{\left|w_{p}\right|,\left|\frac{w_{a}}{p}\right|\right\} \leq 1, \forall \omega$.

This analysis provides insight into the performance vs robustness tradeoff, but it is clear that we need to include a weighting function on the control signals to avoid the singularity of infinite-gain control. Referring back to Figure 2 , the perturbed closed-loop transfer function from the reference to the control signal (SISO case) is given by:

$T_{r u}:=r \mapsto u=w_{u} q\left(1+q w_{a} \Delta_{a}\right)^{-1}$.

The actuator constraint $\left|T_{r u}\right| \leq 1$ can be expressed as: $\left|q w_{u}\right| \leq\left|\left(1+q w_{a} \Delta_{a}\right)^{-1}\right|, \forall\left|\Delta_{a}\right|<1$. Note that normally the relative uncertainty is below $100 \%$, i.e., $\frac{\left|w_{a}\right|}{|p|}<1$, and from the above robust stability constraint $\left|q_{1}\right|<\left|\frac{p}{w_{a}}\right|$, we obtain $\left|q w_{a}\right|=\left|q_{1} \frac{w_{a}}{p}\right|<1$. Thus, the tightest constraint is: $\left|q w_{u}\right| \leq 1-\left|q w_{a}\right|$. Therefore, the resulting constraint on the $\mathrm{IMC}$ filter is given by:

$$
|q| \leq \frac{1}{\left|w_{u}\right|+\left|w_{a}\right|}
$$

Including this constraint in Theorem 1, we get the main result providing a frequency response characterization of an optimal IMC filter in Theorem 2 (also found in [10].)

\section{Theorem 2:}

$$
\begin{aligned}
& \mu_{\Gamma}[g]=\min _{q_{1} \in \mathbb{C},\left|q_{1}\right| \leq \frac{|p|}{\left|w_{u}\right|+\left|w_{a}\right|}}|\underbrace{\frac{w_{a}}{p}}_{\gamma}|\left|q_{1}\right|+\underbrace{\left|w_{p}\right|}_{\beta}\left|1-q_{1}\right|=
\end{aligned}
$$

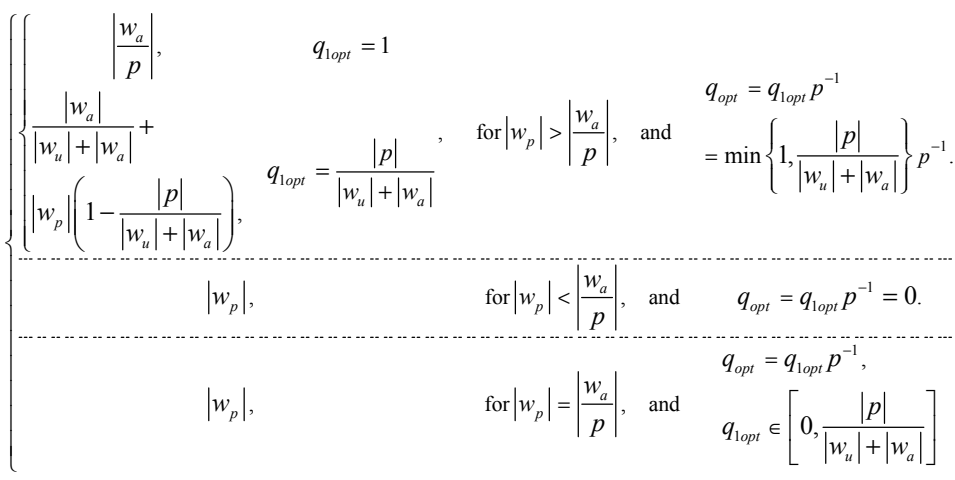

Where $q_{\text {opt }}$ is the optimal IMC filter that minimizes $\mu_{\Gamma}[g]$ while satisfying the actuator constraint. Theorem 2 allows us to draw some additional interesting conclusions as guidelines for controller design and on-line tuning:

- If the additive uncertainty level $\left|w_{a}\right|$ and/or the control weight $\left|w_{u}\right|$ is increased, $q_{1}$ has to decrease for the best tradeoff.

- The robust performance level degrades linearly, i.e., $\mu_{\Gamma}[g]$ increases, with an increase in $\left|w_{p}\right|$.

Also note that all remarks pertaining to Theorem 1 still hold true here.

\section{An LMI Approach to Designing Robust Tunable Controllers}

Linear Matrix Inequalities and associated LMI techniques have emerged as powerful design tools in areas ranging from control engineering to system identification and structural design [9]. Three factors make LMI techniques appealing:

- a variety of design specifications and constraints can be expressed as LMIs,

- once formulated in terms of LMIs, a problem can be solved exactly by efficient convex optimization algorithms (the 'LMI solvers'),

- while most problems with multiple constraints or objectives lack analytical solutions in terms of matrix equations, they often remain tractable in the LMI framework. This makes LMI-based design a valuable alternative to classical 'analytical' methods.

Theorem 2 provides a characterization of the frequency response of an optimal IMC filter $q_{\text {opt }}$. The implementation procedure is to find a stable transfer function $q_{d s n}(s)$ that fits the optimal frequency response. 
A frequency-by-frequency optimization problem can thus be set up as follows:

$$
\min _{q_{d s n} \in H_{\infty}}\left\|q_{d s n}(j \omega)-q_{o p t}(\omega)\right\|
$$

Since we have the magnitude and phase of $q_{\text {opt }}$ at each frequency point, we can treat this optimal magnitude and phase pair as frequency-response data points. Thus, along the frequency grid, the optimization problem (10) can be solved as a matrix norm inequality problem. Next, we set up an equivalent LMI problem to get the desired filter.

\subsection{Setup of the LMI problem}

Given the nominal plant model and the three weighting functions, $q_{\text {opt }}(\omega)$ is first computed using Theorem 2 over a chosen frequency grid. For each frequency $\omega_{k}, k=1, \ldots, N$, the optimization problem (10) becomes: $\min _{q_{d k n} \in H_{\infty}} \rho$, such that

$$
\left[\begin{array}{cc}
\rho^{2} & {\left[q_{d s n}\left(j \omega_{k}\right)-q_{o p t}\left(\omega_{k}\right)\right]^{*}} \\
{\left[q_{d s n}\left(j \omega_{k}\right)-q_{o p t}\left(\omega_{k}\right)\right]} & 1
\end{array}\right]>0
$$

Following [4], we restrict $q_{d s n}$ to the space $\mathcal{S}$ of $L^{\text {th }}$ order discrete-time, real finite impulse response filters. In order to do this, we use a sampling period $T_{s}$ to map the continuous frequency points to discrete points over the range $\Omega_{i} \in[0, \pi]$ using the bilinear transformation. We have:

$$
q_{d s n}\left(j \Omega_{k}\right)=\sum_{i=0}^{L} q_{i} e^{-j \Omega_{k} n}, k=1, \ldots, N .
$$

Note that one could use an FIR filter length of $2 N$ and directly get its coefficients from the set of $2 N$ real linear equations obtained by evaluating polynomial $q_{d s n}\left(z^{-1}\right)$ at the $N$ frequencies. However, although this technique provides perfect interpolation, and notwithstanding the increased filter order, it has the tendency of introducing significant oscillations in the frequency response of the filter. Thus a lower order filter is desirable. Then, the optimization problem (11) is transformed into the following convex LMI problem with complex matrices:

$\min _{q_{d s n} \in \mathcal{S}} \rho^{2}$, such that

$$
\begin{aligned}
& {\left[\begin{array}{cc}
\rho^{2} & {\left[q_{d s n}\left(j \Omega_{k}\right)-q_{o p t}\left(\Omega_{k}\right)\right]^{*}} \\
{\left[q_{d s n}\left(j \Omega_{k}\right)-q_{o p t}\left(\Omega_{k}\right)\right]} & 1
\end{array}\right]>0,} \\
& k=1, \ldots, N
\end{aligned}
$$

where $q_{d s n}\left(j \Omega_{k}\right)=\sum_{i=0}^{L} q_{i} e^{-j \Omega_{k} n}, k=1, \ldots, N$
Recall that the inverse DFT yields a periodic filter $q_{d s n}[n]$, so we have to add this constraint to the LMI problem. Suppose $q_{d s n}[n]$ is periodic, its DFT $q_{d s n}\left(j \Omega_{k}\right)$ is a complex

sequence: $q_{d s n}\left(j \Omega_{k}\right)=q_{r e}\left(j \Omega_{k}\right)+j q_{i m}\left(j \Omega_{k}\right)$. By the Hilbert transform relationship between the real part and imaginary part of $q_{d s n}\left(j \Omega_{k}\right)$ [7], we have for $k=1, \ldots, N$ :

$j q_{i m}\left(j \Omega_{k}\right)=\frac{1}{N} \sum_{m=0}^{N-1} V(k-m) q_{r e}\left(j \Omega_{m}\right)$,

where

$V(k):=\left\{\begin{array}{ccc}-j 2 \cot (\pi k / N), & k & \text { odd } \\ 0, & k & \text { even }\end{array}, 0 \leq k \leq N-1\right.$

We add this equality constraint to the LMIP. Then the optimization problem becomes:

$$
\begin{aligned}
& \min _{q_{d s n} \in \mathcal{S}} \rho^{2}, \text { such that } \\
& {\left[\begin{array}{cc}
\rho^{2} & {\left[q_{d s n}\left(j \Omega_{k}\right)-q_{\text {opt }}\left(\Omega_{k}\right)\right]^{*}}
\end{array}\right]>0,} \\
& {\left[q_{d s n}\left(j \Omega_{k}\right)-q_{\text {opt }}\left(\Omega_{k}\right)\right]} \\
& k=1, \ldots, N \\
& j q_{\text {im }}\left(j \Omega_{k}\right)=\frac{1}{N}\left\{\sum_{m=0}^{N / 2} V(k-m) q_{r e}\left(j \Omega_{m}\right)\right. \\
& \left.\quad+\sum_{m=N / 2+1}^{N-1} V(k-m) q_{r e}\left(j \Omega_{N-m}\right)\right\}, \quad k=0, \ldots, N / 2
\end{aligned}
$$

\subsection{Numerical example}

YALMIP [11] is an interface to a number of semi-definite solvers, and allows the user to define standard problems (LP,SOCP,SDP) in an intuitive way. Its most important features are: (i) Most linear Matlab ${ }^{\mathrm{TM}}$ operators can be applied to matrix variables in order to define objective functions and constraints (LMIs, second order cone constraints and linear constraints), (ii) It supports most of the available public domain semi-definite solvers [8]. The computing environment used was a dual-CPU Pentium III PC with $256 \mathrm{MB}$ RAM, Windows $2000^{\mathrm{TM}}$ and Matlab ${ }^{\mathrm{TM}}$ 6.2 .

Consider a second-order nominal plant model with undamped natural frequency $\omega_{n} \mathrm{rad} / \mathrm{s}$ and damping ratio $\zeta$ with additive uncertainty:

$$
p=\frac{k_{0} \omega_{n}^{2}}{s^{2}+2 \zeta \omega_{n} s+\omega_{n}^{2}} .
$$

Let us pick $\zeta=0.5, \quad \omega_{n}=100 \mathrm{rd} / \mathrm{s}, \quad k_{0}=3$. The sampling period is set to $T_{s}=0.005 \mathrm{~s}$. Numerically, 
$p=\frac{3 \times 10^{4}}{s^{2}+100 s+10^{4}}$, and the weighting functions are $w_{a}=\frac{15}{s+110}, w_{u}=\frac{0.5(s+1)}{0.01 s+1}, w_{p}=\frac{0.5}{0.01 s+1}$.

Figure 4 shows the fit of the designed $25^{\text {th }}$-order FIR IMC filter to the optimal frequency response.
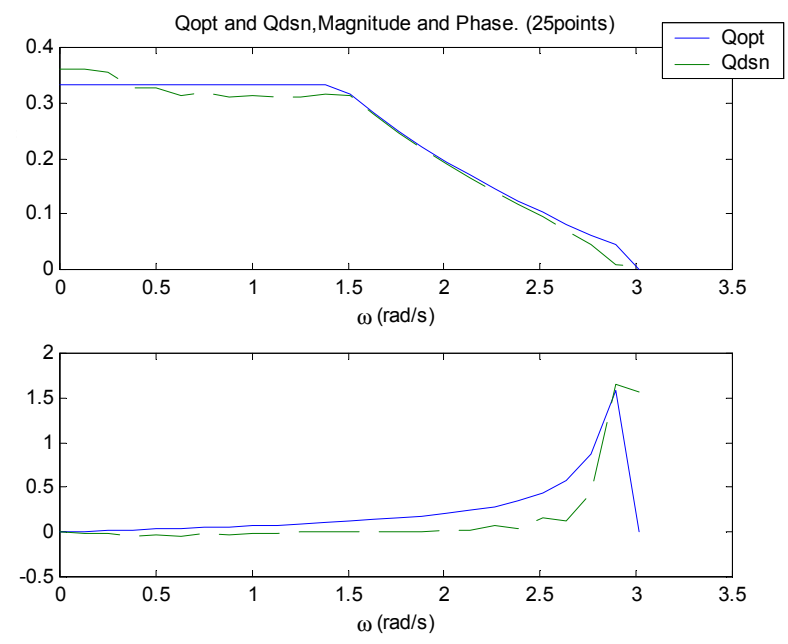

Figure 4: Numerical result of the designed IMC filter using LMI approach. Number of frequency points:25, solver time: $20 s$

Figure 5 shows a plot of the structured singular value computed using Theorem 2 with the $25^{\text {th }}$-order IMC filter. Clearly this design meets the performance robustness specification.

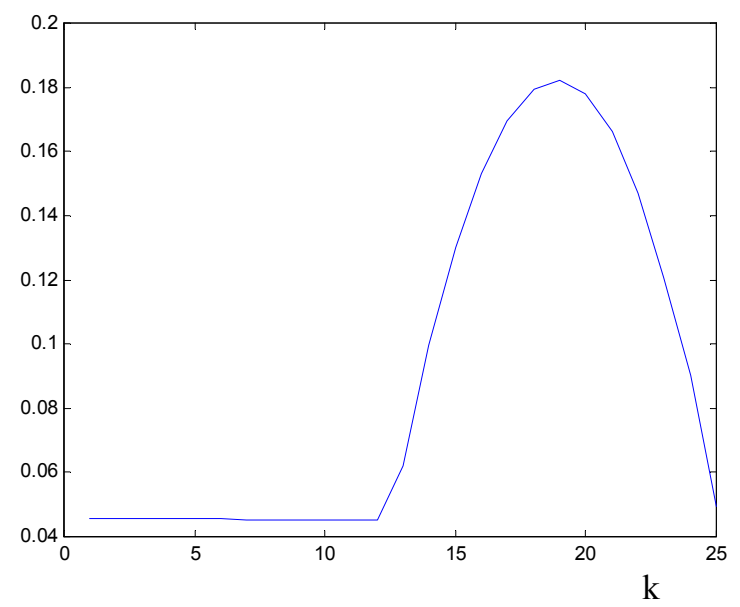

Figure 5: Plot of $\mu_{\Gamma}[g]$ vs frequency point index computed using Theorem 2 with the designed IMC filter. Number of frequency points: 25

\section{Conclusion}

The numerical results shows that an FIR IMC filter $q_{d s n}$ with a reasonable fit on the optimal frequency response $q_{\text {opt }}(\omega)$ can be obtained in less than a minute using an LMI solver on a PC.

Furthermore, Theorem 2 provides a very quick and simple way to compute $\mu_{\Gamma}[g]$. Our proposed approach to robust tunable control for SISO plants with additive uncertainty leads to the possibility for the plant engineer to tune the controller on-line by directly changing either the uncertainty, the performance, or the actuator weighting functions, as the need arises. Future research will address the robust tunable control problem for unstable and multivariable plants with LFT uncertainty models.

\section{References}

[1] K. Zhou and J. C. Doyle, Essentials of Robust Control. Prentice Hall, New Jersey, 1998.

[2] M. Morari and E. Zafirious, Robust Process Control. PTR Prentice Hall, New Jersey, 1989.

[3] B. Zhu, H. S. Lee, L. Guo and M. Tomizuka, Robust Tuning of Fixed-Structure Controller for Disk Drives Using Statistical Model and MOGA. Proc. 2001 American Control Conference, June 2001, pp. 2773-2778.

[4] K.-Y. Tsai, and H. A. Hindi, DQIT: $\mu$ synthesis without D-scale fitting. Proc. 2002 American Control Conference, May 2002, pp. 493-498.

[5] J.G. Owen and G. Zames, Duality Theory of Robust Disturbance Attenuation. Automatica, May 1993.

[6] J. G. Owen, Performance Optimization of Highly Uncertain Systems in H-Infinity. Ph.D Thesis, Department of Electrical \& Computer Engineering, McGill University, January 1993.

[7] A. V. Oppenheim and R. W. Shafer, Discrete-time Signal Processing, Prentice Hall, 1989.

[8] L. Vandenberghe and S. Boyd, SP: Software for Semidefinite Programming . Stanford University, 1994.

[9] S. Boyd, L. El Ghaoui, E. Feron, and V. Balakrishnan, Linear Matrix Inequalities in System and Control Theory. SIAM, 1994.

[10] B. Boulet, Y. Duan and H. Michalska, Some Preliminary Results for IMC-Based Robust Tunable Control. Proc. 2002 American Control Conference, May 2002, pp. 4894-4895.

[11] J. Lofberg, Yalmip, another parser of LMI tool box. http://www.control.isy.liu.se/ johanl/. 